\title{
Decidable Properties for Regular Cellular Automata
}

\author{
Pietro Di Lena \\ Department of Computer Science, University of Bologna, Mura Anteo Zamboni 7, \\ 40127 Bologna, Italy. dilena@cs . unibo. it
}

\begin{abstract}
We investigate decidable properties for regular cellular automata. In particular, we show that regularity itself is an undecidable property and that nilpotency, equicontinuity and positively expansiveness became decidable if we restrict to regular cellular automata.
\end{abstract}

\section{Introduction}

Cellular Automata (CA) are often used as a simple model for complex systems. They were introduced by Von Neumann in the forties as a model of selfreproductive biological systems [16]. Mathematical theory of CA was developed later by Hedlund in the context of symbolic dynamics [7].

To a cellular automaton one associates the shift spaces generated by the evolution of the automaton on suitable partitions of the configuration space. Adopting Kürka's terminolgy we call column subshifts this kind of shift spaces (see [12] chapter 5). A general approach to the study of a cellular automaton is to study the complexity of its column subshifts (see $[5,13,10]$ ).

Regularity has been introduced by Kůrka for general dynamical systems [14]. A CA is regular if every column subshift is sofic, i.e. if the language of every column subshift is regular. Kurrka classified $\mathrm{CA}$ according to the complexity of column subshift languages [13]. In Kůrka's classification the main distiction is whether the cellular automaton is regular or not. He compared language classification with two other famous CA classifications such as equicontinuity and attractor classification.

In this paper we study the decidability of topological properties for CA. In particular, we show that regularity is not a decidable property (Theorem 7) which implies that the membership in Kưrka's language classes is undecidable. In contrast, we show that some topological properties which are in general undecidable become decidable if we restrict to the class of regular $\mathrm{CA}$. For instance, we show that for regular CA nilpotency, equicontinuity and positively expansiveness are decidable properties (Theorem 6). Moreover, we provide an answer to a question raised in [3] showing that the topological entropy is computable for one-sided regular CA (Theorem 5).

The paper is organized as follows. Section 2 is devoted to the introduction of the notation and general definitions while Section 3 contains our results.

Please use the following format when citing this chapter:

Di Lena, P., 2006, in International Federation for Information Processing, Volume 209, Fourth IFIP International Conference on Theoretical Computer Science-TCS 2006, eds. Navarro, G., Bertossi, L., Kohayakwa, Y., (Boston: Springer), pp. 185-196. 


\section{Notations and Definitions}

\subsection{Shift Spaces and representations of Sofic Shifts}

Let $A=\left\{a_{1}, \ldots, a_{n}\right\}$ be a finite alphabet, $n>1$. For any $k>0, w_{1} w_{2} \ldots w_{k} \in A^{k}$ is a finite sequence of elements of $A$. The sets $A^{\mathbb{Z}}$ and $A^{\mathbb{N}}$ are respectively the set of doubly infinite sequences $\left(x_{i}\right)_{i \in \mathbb{Z}}$ and mono infinite sequences $\left(x_{i}\right)_{i \in \mathbb{N}}$ where $x_{i} \in A$. Let $x \in A^{\mathbb{Z}}$, for any integer interval $[i, j], x_{[i, j]} \in A^{j-i+1}$ is the finite subword $x_{i} x_{i+1} \ldots x_{j}$ of $x$.

Define the metric $d$ on $A^{\mathbb{Z}}$ by $d(x, y)=\Sigma_{i \in \mathbb{Z}} \frac{d_{i}\left(x_{i}, y_{i}\right)}{2^{|i|}}$ where $d_{i}\left(x_{i}, y_{i}\right)=1$ if $x_{i}=y_{i}$ and $d_{i}\left(x_{i}, y_{i}\right)=0$ otherwise. The set $A^{\mathbb{Z}}$ endowed with metric $d$ is a compact metric space. A dynamical system is a pair $(X, F)$ where $F: X \rightarrow X$ is a continuous function and $X$ is a compact metrizable space. The shift map $\sigma: A^{\mathbb{Z}} \rightarrow A^{\mathbb{Z}}$, defined by $\sigma(x)_{i}=x_{i+1}$, is an homeomorphism of the compact metric space $A^{\mathbb{Z}}$. The dynamical system $\left(A^{\mathbb{Z}}, \sigma\right)$ is called full n-shift or simply full shift.

A shift space or subshift $(X, \sigma)$ is a closed shift invariant subset of $A^{\mathbb{Z}}$ endowed with $\sigma$. The shift dynamical system $(X, \sigma)$ is called one-sided if $X \subseteq A^{\mathbb{N}}$. In general, we denote the subshift $(X, \sigma)$ simply with $X$.

Let denote with $\mathcal{B}_{k}(X)=\left\{x \in A^{k} \mid \exists y \in X, \exists i \in \mathbb{Z}, y_{[i, i+k-1]}=x\right\}$ the set of allowed $k$-blocks of the subshift $X, k>0$. The language associated to a subshift $X$ is denoted with $\mathcal{L}(X)=\Sigma_{i \in \mathbb{N}} \mathcal{B}_{k}(X)$. Any subshift is completely determined by its language (see [15]). The language of a subshift $X$ is:

1. factorial: if $x y z \in \mathcal{L}(X)$ then $y \in \mathcal{L}(X)$.

2. extendable: $\forall x \in \mathcal{L}(X), \exists y \in \mathcal{L}(X)$ such that $x y \in \mathcal{L}(X)$.

The language $\mathcal{L}(X)$ of a subshift $X$ is bounded periodic if there exists integers $m \geq 0, n>0$ such that $\forall x \in \mathcal{L}(X)$ and $\forall i \geq m, x_{i}=x_{i+n}$.

A factor map $F:(X, \sigma) \rightarrow(Y, \sigma)$ is a continuous and $\sigma$-commuting function, i.e. $F \circ \sigma=\sigma \circ F$. If $F$ is onto (or surjective), $X$ is called extension of $Y$ and $Y$ is called factor of $X$. If $F$ is biiective, it is a topological conjugacy and $X, Y$ are said to be topologically conjugated shift spaces.

A subshift is sofic if it can be represented by means of a labeled graph. We review the representation of a sofic shift as vertex shift of a labeled graph. A labeled graph $\mathcal{G}=(V, E, \zeta)$ consists of a set of vertices $V$, a set of edges $E$ and a labeling function $\zeta: V \rightarrow A$ which assigns to each vertex $v \in V$ a symbol from a finite alphabet $A$. Each edge $e \in E$ identifies an initial vertex $i(e) \in V$ and a terminal vertex $t(e) \in V$. We denote the existence of an edge between vertices $v, v^{\prime} \in V$ by $v \rightarrow v^{\prime}$. Every sofic shift can be represented as the set of (mono or doubly) infinite sequences generated by the labels of vertices of a labeled graph. That is, the labeled graph $\mathcal{G}=(V, E, \zeta)$, with $\zeta: V \rightarrow A$, represents the (two-sided) sofic shift

$$
S_{\mathcal{G}}=\left\{x \in A^{\mathbb{Z}} \mid \exists\left(v_{i}\right)_{i \in \mathbb{Z}} \in V^{\mathbb{Z}}, v_{i} \rightarrow v_{i+1}, \zeta\left(v_{i}\right)=x_{i}, i \in \mathbb{Z}\right\} .
$$


The topological entropy $h(X)=\lim _{n \rightarrow \infty} \log \left|\mathcal{B}_{n}(X)\right| / n$ of a shift space $X$ is a measure of the complexity of $X$. While the topological entropy is not computable for general subshifts, it is for sofic shifts (see [15]).

The language of a sofic shift is denoted as regular in the context of formal language theory (see [9] for an introduction). The class of regular languages is the class of languages which can be recognized by a deterministic finite state automaton (DFA). Formally, a DFA is a 5-tuple $\left(Q, A, \delta, q_{0}, F\right)$ where $Q$ is a finite set of states, $F \subseteq Q$ is the set of accepting states, $q_{0} \in Q$ is the initial state, $A$ is a finite alphabet and $\delta: Q \times A \rightarrow Q$ is a partial transition function (i.e. it can be defined only on a subset of $Q \times A$ ). The language represented by a DFA is the set of words generated by following a path starting from the initial state and ending to an accepting state.

For every regular language there exists an unique smallest DFA, where smallest refers to the number of states. In general, most of the questions concerning regular languages are algorithmically decidable. In particular, it is decidable if two distinct DFA represent the same language.

From a DFA representing the language of a sofic shift $S$ it is possible to derive a labeled graph presentation of $S$ in the following way:

1. the set of vertices $V$ consists of the pairs $(q, a) \in Q \times A$ s.t. $\delta(q, a) \in Q$.

2. there exists an edge $(q, a) \rightarrow\left(q^{\prime}, a^{\prime}\right),(q, a),\left(q^{\prime}, a^{\prime}\right) \in V$, if $\delta(q, a)=q^{\prime}$

3. $\forall v=(q, a) \in V, \zeta(v)=a$.

\subsection{Cellular Automata}

A cellular automaton is a dynamical system $\left(A^{\mathbb{Z}}, F\right)$ where $A$ is a finite alphabet and $F$ is a $\sigma$-commuting, continuous function. $\left(A^{\mathbb{Z}}, F\right)$ is generally identified by a block mapping $f: A^{2 r+1} \rightarrow A$ such that $F(x)_{i}=f\left(x_{[i-r, i+r]}\right), i \in \mathbb{Z}$. According to Curtis-Hedlund-Lyndon Theorem [7], the whole class of continuous and $\sigma$ commuting functions between shift spaces arises in this way.

We refer to $f$ and $r$ respectively as local rule and radius of the CA.

A CA is one-sided, if the local rule is of the form $f: A^{r+1} \rightarrow A$ where $\forall x \in A^{\mathbb{Z}}, i \in \mathbb{Z}, F(x)_{i}=f\left(x_{[i, i+r]}\right)$. A one-sided CA is usually denoted with $\left(A^{\mathbb{N}}, F\right)$.

We recall the definition of some topological properties of CA. Let $d$ denote the metric on $A^{\mathbb{Z}}$ defined in Section 2.1 .

Definition 1. Let $\left(A^{\mathbb{Z}}, F\right)$ be a $C A$.

1. $\left(A^{\mathbb{Z}}, F\right)$ is nilpotent if

$$
\exists N>0, \exists x \in A^{\mathbb{Z}}, \sigma(x)=x, \text { s.t. } \forall n \geq N, F^{n}\left(A^{\mathbb{Z}}\right)=x .
$$

2. $\left(A^{\mathbb{Z}}, F\right)$ is equicontinuous at $x \in A^{\mathbb{Z}}$ if

$$
\forall \epsilon>0, \exists \delta>0 \text { s.t. } \forall y \in A^{\mathbb{Z}}, d(x, y)<\delta, \exists n>0 \text { s.t. } d\left(F^{n}(x), F^{n}(y)\right)<\epsilon .
$$

3. $\left(A^{\mathbb{Z}}, F\right)$ is equicontinuous if $\forall x \in A^{\mathbb{Z}},\left(A^{\mathbb{Z}}, F\right)$ is equicontinuous at $x$. 
4. $\left(A^{\mathbb{Z}}, F\right)$ is almost equicontinuous if $\exists x \in A^{\mathbb{Z}}$ s.t. $\left(A^{\mathbb{Z}}, F\right)$ is equicont. at $x$.

5. $\left(A^{\mathbb{Z}}, F\right)$ is sensitive if

$$
\begin{gathered}
\exists \epsilon>0 \text { s.t. } \forall x \in A^{\mathbb{Z}}, \forall \delta>0, \exists y \in A^{\mathbb{Z}}, d(x, y)<\delta, \exists n>0 \text { s.t. } \\
d\left(F^{n}(x), F^{n}(y)\right) \geq \epsilon .
\end{gathered}
$$

5. $\left(A^{\mathbb{Z}}, F\right)$ is positively expansive if

$$
\exists \epsilon>0 \text { s.t. } \forall x, y \in A^{\mathbb{Z}}, x \neq y, \exists n>0 \text { s.t. } d\left(F^{n}(x), F^{n}(y)\right) \geq \epsilon .
$$

Kari showed that nilpotency is an undecidable property [11]. In [4], Durand et al. showed that equicontinuity, almost equicontinuity and sensitivity are undecidable properties. Actually, it is unknown if positively expansiveness is or not a decidable property.

Definition 2. (Column subshift) Let $\left(A^{\mathbb{Z}}, F\right)$ be a $C A$. For $k>0$ let

$$
\Sigma_{k}=\left\{x \in\left(A^{k}\right)^{\mathbb{N}} \mid \exists y \in A^{\mathbb{Z}}: F^{i}(y)_{[0, k)}=x_{i}, i \in \mathbb{N}\right\}
$$

denote the column subshift of width $k$ associated to $\left(A^{\mathbb{Z}}, F\right)$.

Gilman noticed that the language of a column subshift is always contextsensitive [6]. Kürka classified cellular automata according to the complexity of column subshifts languages [13].

Definition 3. (Bounded periodic $C A)\left(A^{\mathbb{Z}}, F\right)$ is bounded periodic if $\forall t>0$, $\mathcal{L}\left(\Sigma_{t}\right)$ is a bounded periodic language.

Definition 4. (Regular $C A)\left(A^{\mathbb{Z}}, F\right)$ is regular if $\forall t>0, \mathcal{L}\left(\Sigma_{t}\right)$ is a regular language (or, equivalently, if $\Sigma_{t}$ is sofic shift).

Definition 5. (Kuirka's Language classification) Every cellular automaton falls exactly in one of the following classes.

L1. Bounded periodic.

L2. Regular not bounded periodic.

L3. Not regular.

Class $L 1$ coincide with the class of equicontinuous CA [13]. Thus the membership in $L 1$ is undecidable while it was unknown if it is for $L 2, L 3$.

The topological entropy $H(F)=\lim _{k \rightarrow \infty} h\left(\Sigma_{k}\right)$ of $\left(A^{\mathbb{Z}}, F\right)$ is a measure of the complexity of the dynamics of $\left(A^{\mathbb{Z}}, F\right)$. The problem of computing or even approximating the topological entropy of $\mathrm{CA}$ has been shown to be in general not algorithmically computable [8]. The topological entropy of one-sided CA has a simpler characterization than the general case (see [2]).

Theorem 1. Let $\left(A^{\mathbb{N}}, F\right)$ be a $C A$ with radius $r$. Then $H(F)=h\left(\Sigma_{r}\right)$. 


\section{Results}

In this section we investigate decidable properties of regular CA. Most of our effort will be devoted to show that if $S \subseteq\left(A^{2 r+1}\right)^{\mathbb{N}}$ is a sofic shift and $\left(A^{\mathbb{Z}}, F\right)$ is a CA with radius $r$, it is possible to decide whether $S=\Sigma_{2 r+1}$ (Theorem 3 ). This strong result has a lot of consequences. The most relevant one is that for regular CA it is possible to compute column subshifts of every given width (Theorem 4). The (dynamical) complexity of a CA is strictly related to the complexity of column subshifts languages. Actually we show that, thanks to the computability property, it is possible to decide if a regular CA is nilpotent, equicontinuous or positively expansive (Theorem 6). Moreover, it turns also out, that it is possible to compute the topological entropy for one-sided regular CA (Theorem 5). The negative consequence of computability/decidability results is that regularity itself is an undecidable property (Theorem 7).

In order to show our fundamental decidability result (Theorem 3) we need to define the concept of cellular automaton extension of a sofic shift and to show some basic properties.

Definition 6. Let $\left(A^{\mathbb{Z}}, F\right)$ be a $C A$ with radius $r$. Let $\mathcal{G}=(V, E, \zeta)$ be a labeled graph with $\zeta: V \rightarrow A^{2 r+1}$. For $t>0$, let the $(\mathrm{F}, \mathrm{t})$-extension of $\mathcal{G}$ be the labeled graph $\mathcal{G}_{(F, t)}=\left(V_{t}, E_{t}, \zeta_{t}\right)$, with $\zeta_{t}: V_{t} \rightarrow A^{2 r+t}$, defined in the following way (see figure 1):

- vertex set:

$$
V_{t}=\left\{\left(v_{1}, . ., v_{t}\right) \in V^{t} \mid \exists a \in A^{2 r+t}, \zeta\left(v_{i}\right)=a_{[i, 2 r+i]}, 1 \leq i \leq t\right\}
$$

- edge set:

$$
E_{t}=\left\{\left(e_{1}, . ., e_{t}\right) \in E^{t} \mid \exists v, v^{\prime} \in V_{t}, i\left(e_{j}\right)=v_{j}, t\left(e_{j}\right)=v_{j}^{\prime}, f\left(\zeta\left(v_{j}\right)\right)=\zeta\left(v_{j}^{\prime}\right)_{r+1}\right\}
$$

- labeling function:

$$
\forall v=\left(v_{1}, \ldots, v_{t}\right) \in V_{t}, \zeta_{t}(v)=\text { a where } a_{[i, 2 r+i]}=\zeta\left(v_{i}\right), 1 \leq i \leq t .
$$

Definition 7. Let $\left(A^{\mathbb{Z}}, F\right)$ be a $C A$. Let $t>0, k>1$ and let $a, b \in \mathcal{B}_{t}\left(\Sigma_{k}\right)$ such that $a=a_{1} \ldots a_{k}, b=b_{1} \ldots b_{k}$ where $a_{i}, b_{i} \in A^{t}$ and $a_{i+1}=b_{i}, 1 \leq i<k$. Then, we say that $x, y$ are compatible blocks and we denote with $a \odot b=a_{1} \ldots a_{k} b_{k}$ their overlapping concatenation.

Moreover, let $x, y \in \Sigma_{k}$ such that $x=x_{1} \ldots x_{k}, y=y_{1} \ldots y_{k}$ where $x_{i}, y_{i} \in$ $A^{\mathbb{N}}$ and $x_{i+1}=y_{i}, 1 \leq i<k$. We say that $x, y$ are compatible sequences and, abusing the notation, we denote with $x \odot y=x_{1} \ldots x_{k} y_{k}$ their overlapping concatenation.

The following two lemmas will be used extensively. 


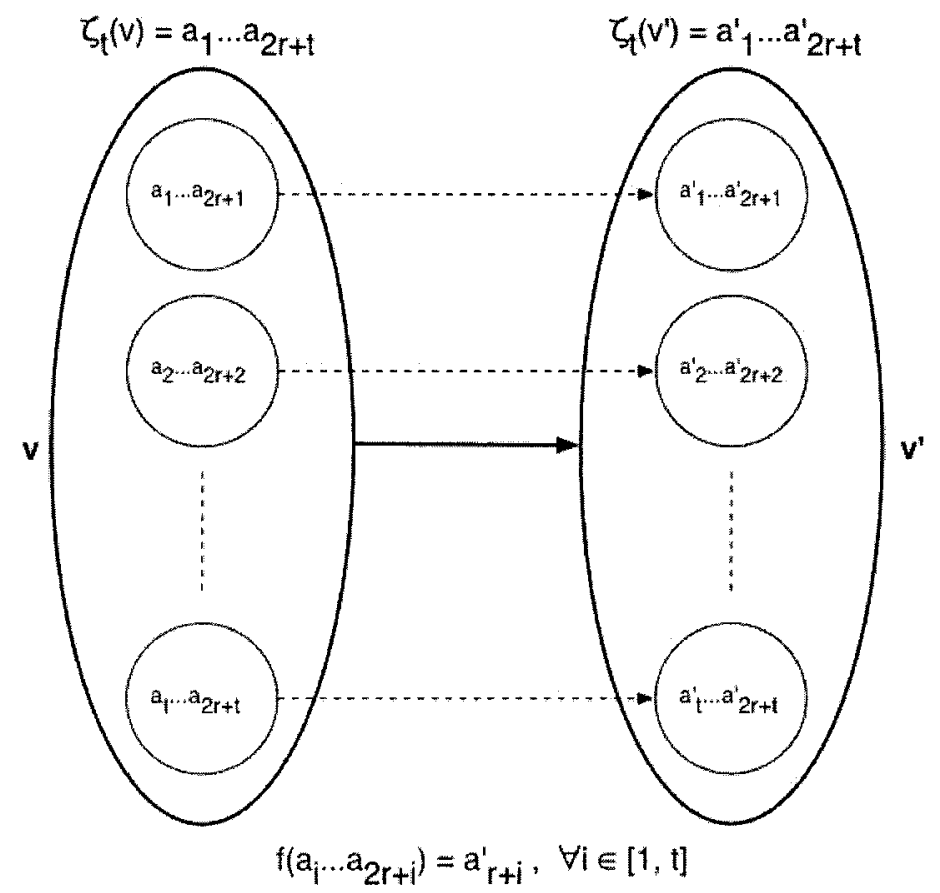

Fig. 1. A legal edge $v \rightarrow v^{\prime}$ of an $(F, t)$-extended graph $\mathcal{G}_{(F, t)}$.

Lemma 1. Let $\left(A^{\mathbb{Z}}, F\right)$ be a $C A$ with radius $r$. Let $t>0$ and let $a, b \in$ $\mathcal{B}_{t}\left(\Sigma_{2 r+1}\right)$ be compatible blocks. Then $a \odot b \in \mathcal{B}_{t}\left(\Sigma_{2 r+2}\right)$.

Proof. Let $a=a_{1} \ldots a_{t}$ where $a_{1}, \ldots, a_{t} \in A^{2 r+1}$ and let $x \in A^{\mathbb{Z}}$ such that $F^{i}(x)_{[0,2 r]}=a_{i+1}, 0 \leq i<t$. Moreover, let $b=b_{1} \ldots b_{t}$ where $b_{1}, \ldots, b_{t} \in A^{2 r+1}$ and let $y \in A^{\mathbb{Z}}$ such that $F^{i}(y)_{[1,2 r+1]}=b_{i+1}, 0 \leq i<t$. Let $z \in A^{\mathbb{Z}}$ be such that $z_{(-\infty, 2 r]}=x_{(-\infty, 2 r]}, z_{[1, \infty)}=y_{[1, \infty)}$ and let $a \odot b=c_{1} \ldots c_{t}$ where $c_{1}, \ldots, c_{t} \in A^{2 r+2}$. Then it is easy to check that $F^{i}(z)_{[0,2 r+1]}=c_{i+1}, 0 \leq i<t$ which implies that $a \odot b \in B_{t}\left(\Sigma_{2 r+2}\right)$.

Lemma 2. Let $\left(A^{\mathbb{Z}}, F\right)$ be a $C A$ with radius $r$. Let $S \subseteq\left(A^{2 r+1}\right)^{\mathbb{N}}$ be a sofic shift and let $\mathcal{G}$ be a labeled graph presentation of $S$. Let $x, y \in S_{\mathcal{G}_{(F, 1)}}$ be compatible sequences. Then $x \odot y \in S_{\mathcal{G}_{(F, 2)}}$.

Proof. Since, by hypothesis, $x=\left(x_{i}\right)_{i \in \mathbb{N}}, y=\left(y_{i}\right)_{i \in \mathbb{N}} \in S_{\mathcal{G}_{(F, 1)}}$, there exist two paths $u_{1} \rightarrow u_{2} \rightarrow \ldots$ and $v_{1} \rightarrow v_{2} \rightarrow \ldots$ in $\mathcal{G}$ such that $\zeta\left(u_{i}\right)=x_{i}$ and $\zeta\left(v_{i}\right)=y_{i}$, $i \in \mathbb{N}$. Then, $\left(u_{1}, v_{1}\right) \rightarrow\left(u_{2}, v_{2}\right) \rightarrow \ldots$ is a legal path in $\mathcal{G}_{(F, 2)}$ which implies that $x \odot y \in S_{\mathcal{G}_{(F, 2)}}$.

The following proposition shows that the sofic shift presented by the $(F, t)$ extension $\mathcal{G}_{(F, t)}$ of a labeled graph $\mathcal{G}$ doesn't depend on $\mathcal{G}$ but only on the sofic shift presented by $\mathcal{G}$. 
Proposition 1. Let $\left(A^{\mathbb{Z}}, F\right)$ be a $C A$ with radius $r$ and let $\mathcal{G}, \mathcal{G}^{\prime}$ be two distinct labeled graph presentations of the same sofic shift $S=S_{\mathcal{G}}=S_{\mathcal{G}^{\prime}} \subseteq\left(A^{2 r+1}\right)^{\mathbb{N}}$. Then, for any $t>0, S_{\mathcal{G}_{(F, t)}}=S_{\mathcal{G}_{(F, t)}^{\prime}}$.

Proof. We show that $S_{\mathcal{G}_{(F, t)}} \subseteq S_{\mathcal{G}_{(F, t)}^{\prime}}$. The proof for the converse inclusion can be obtained by exchanging $\mathcal{G}$ with $\mathcal{G}^{\prime}$.

First of all, note that, by definition of $(F, 1)$-extension, $S_{\mathcal{G}_{(F, 1)}}=S_{\mathcal{G}_{(F, 1)}^{\prime}}$. Let $x \in S_{\mathcal{G}_{(F, t)}}$ and let $x_{1}, \ldots, x_{t} \in S$ such that $x=x_{1} \odot \ldots \odot x_{t}$. Then, $x_{1}, \ldots, x_{t} \in$ $S_{\mathcal{G}_{(F, 1)}^{\prime}}$ and, by Lemma 2 , it follows that $x \in S_{\mathcal{G}_{(F, t)}^{\prime}}$.

Thanks to Proposition 1 we can refer directly to the extension of a sofic shift $S$ rather than to the extension of a labeled graph presentation of $S$.

Definition 8. Let $\left(A^{\mathbb{Z}}, F\right)$ be a $C A$ with radius $r$. Let $S \subseteq\left(A^{2 r+1}\right)^{\mathbb{N}}$ be a sofic shift and let $\mathcal{G}$ be a labeled graph presentation of $S$. For $t>0$, let denote with $S_{(F, t)}=S_{\mathcal{G}_{(F, t)}}$ the $(\mathrm{F}, \mathrm{t})$-extension of the sofic shift $S$.

We now show some useful properties of the $(F, t)$-extensions of sofic shifts.

Lemma 3. Let $\left(A^{\mathbb{Z}}, F\right)$ be a $C A$ with radius $r$. Let $S \subseteq\left(A^{2 r+1}\right)^{\mathbb{N}}$ be a sofic shift. Then $\forall t>0$,

a. if $\Sigma_{2 r+1} \subset S$ then $\Sigma_{2 r+t} \subseteq S_{(F, t)}$,

b. if $\Sigma_{2 r+1}=S$ then $\Sigma_{2 r+t}=S_{(F, t)}$,

c. if $\Sigma_{2 r+1} \supset S$ then $\Sigma_{2 r+t} \supset S_{(F, t)}$.

Proof. a. Let $x \in \Sigma_{2 r+t}$ such that $x=x_{1} \odot . \odot x_{t}$ where $x_{i} \in \Sigma_{2 r+1}, 1 \leq i \leq t$.

Then, $x_{i} \in S_{(F, 1)}, 1 \leq i \leq t$ and, by Lemma $2, x_{1} \odot . \odot x_{t} \in S_{(F, t)}$.

b. By point $a, \Sigma_{2 r+t} \subseteq S_{(F, t)}$, thus we just have to show that $S_{(F, t)} \subseteq \Sigma_{2 r+t}$ or, equivalently, that $\mathcal{L}\left(S_{(F, t)}\right) \subseteq \mathcal{L}\left(\Sigma_{2 r+t}\right)$. Let $k>0$ and let $a \in \mathcal{B}_{k}\left(S_{(F, t)}\right)$. Let $a_{1}, \ldots, a_{t} \in \mathcal{B}_{k}(S)$ be such that $a_{1} \odot \ldots \odot a_{t}=a$. By hypothesis, $a_{1}, \ldots, a_{t} \in$ $\mathcal{B}_{k}\left(\Sigma_{2 r+1}\right)$ then, by Lemma 1 , it follows that $a_{1} \odot \ldots \odot a_{t} \in \mathcal{B}_{k}\left(\Sigma_{2 r+t}\right)$.

c. Since $\Sigma_{2 r+1} \supset S$, appling the same reasoning of point $b$, it is possible to conclude that $\Sigma_{2 r+t} \supseteq S_{(F, t)}$. We have just to show that the inclusion is strict. Since $\Sigma_{2 r+1} \supset S$, there exists a block $b_{1} \in \mathcal{L}\left(\Sigma_{2 r+1}\right)$ such that $b_{1} \notin$ $\mathcal{L}(S)$. Then, let $b \in \mathcal{L}\left(\Sigma_{2 r+t}\right)$ such that $b=b_{1} \odot b_{2} \odot \ldots \odot b_{t}$ for some $b_{2}, \ldots, b_{t} \in \mathcal{L}\left(\Sigma_{2 r+1}\right)$. Trivially, $b \notin \mathcal{L}\left(S_{(F, t)}\right)$.

The following theorem easily follows from Lemma 3 and provides a strong characterization for regular CA. It is a two-sided extension of a theorem proved by Blanchard and Maass for one-sided CA [1].

Theorem 2. Let $\left(A^{\mathbb{Z}}, F\right)$ be a $C A$ with radius $r$. Then $\left(A^{\mathbb{Z}}, F\right)$ is regular if and only if $\Sigma_{2 r+1}$ is a sofic shift.

Proof. The necessary implication is trivial. Then, suppose $\Sigma_{2 r+1}$ is a sofic shift. For every $d<2 r+1, \Sigma_{d}$ is a factor of $\Sigma_{2 r+1}$ then it is a sofic shift. For every $d>2 r+1$, by Lemma 3 point b, $\Sigma_{d}$ can be represented by a labeled graph then it is a sofic shift.

In general, if $\Sigma_{d}$ is a sofic shift for $d<2 r+1$ it is not possible to conclude that the CA is regular (see [10]). 
Definition 9. Let $A$ be a finite alphabet. Let $t \geq 1$ and let $[i, j] \subseteq[1, t]$ be an integer interval. Let

$$
\Phi_{[i, j]}:\left(A^{t}\right)^{\mathbb{N}} \rightarrow\left(A^{j-i+1}\right)^{\mathbb{N}}
$$

denote the projection map induced by the one-block factor map

$$
\varphi_{[i, j]}: A^{t} \rightarrow A^{j-i+1}
$$

defined by $\varphi_{[i, j]}\left(a_{1} \ldots a_{t}\right)=a_{i} a_{i+1} \ldots a_{j}, \forall a_{1} a_{2} \ldots a_{t} \in A^{t}$.

Remark 1. Let $\left(A^{\mathbb{Z}}, F\right)$ be a $\mathrm{CA}$ with radius $r$ and let $\mathcal{G}_{(F, t)}$ be the $(F, t)$ extension of $\mathcal{G}$. Then for every $i \in[1, t], \Phi_{[i, 2 r+i]}\left(S_{\mathcal{G}_{(F, t)}}\right) \subseteq S_{\mathcal{G}}$.

Definition 10. Let $\left(A^{\mathbb{Z}}, F\right)$ be a $C A$ with radius $r$ and let $S \subseteq\left(A^{2 r+1}\right)^{\mathbb{N}}$ be a sofic shift. $S$ is $F$-extendible if

$$
S=\Phi_{[i, 2 r+i]}\left(S_{(F, t)}\right), \forall t>0, \forall i \in[1, t] .
$$

Note that for a sofic shift to be $F$-extendible is a necessary condition in order to be equal to $\Sigma_{2 r+1}$.

Proposition 2. Let $\left(A^{\mathbb{Z}}, F\right)$ be a $C A$ with radius $r$ and let $S \subseteq\left(A^{2 r+1}\right)^{\mathbb{N}}$ be a sofic shift. Then, $S$ is F-extendible iff $S=\Phi_{[1,2 r+1]}\left(S_{(F, 2)}\right)=\Phi_{[2,2 r+2]}\left(S_{(F, 2)}\right)$.

Proof. The necessary implication is trivial. Then, let $S=\Phi_{[1,2 r+1]}\left(S_{(F, 2)}\right)=$ $\Phi_{[2,2 r+2]}\left(S_{(F, 2)}\right)$. Note that this implies $S=S_{(F, 1)}$. Let $t>2$, we have to show that $S=\Phi_{[i, 2 r+i]}\left(S_{(F, t)}\right)$ for $1 \leq i \leq t$. Let $z \in S$ and let $k \in[1, t]$. To reach the proof it is sufficient to show that $z \in \Phi_{[k, 2 r+k]}\left(S_{(F, t)}\right)$. Since $S=\Phi_{[1,2 r+1]}\left(S_{(F, 2)}\right)=\Phi_{[2,2 r+2]}\left(S_{(F, 2)}\right)$, there exists $x_{1}, . ., x_{t-1} \in S_{(F, 2)}$ such that $\Phi_{[2,2 r+2]}\left(x_{i}\right)=\Phi_{[1,2 r+1]}\left(x_{i+1}\right), 1 \leq i<t-1$ and $\Phi_{[2,2 r+2]}\left(x_{k-1}\right)=$ $\Phi_{[1,2 r+1]}\left(x_{k}\right)=z$. Then, $x_{1}, \ldots, x_{t-1}$ are compatible and by Lemma 2 , it follows that $x_{1} \odot \ldots \odot x_{t-1} \in S_{(F, t)}$ and $\Phi_{[k, 2 r+k]}\left(x_{1} \odot \ldots \odot x_{t-1}\right)=z$.

Proposition 3. Let $\left(A^{\mathbb{Z}}, F\right)$ be a $C A$ with radius $r$ and let $S \subseteq\left(A^{2 r+1}\right)^{\mathbb{N}}$ be a sofic shift. Suppose $S$ is $F$-extendible then $S \subseteq \Sigma_{2 r+1}$.

Proof. We prove by induction on $k>0$ that $\mathcal{B}_{k}(S) \subseteq \mathcal{B}_{k}\left(\Sigma_{2 r+1}\right)$.

1. (Base Case) By definition, $\mathcal{B}_{1}(S) \subseteq \mathcal{B}_{1}\left(\Sigma_{2 r+1}\right)=A^{2 r+1}$.

2. (Inductive Case) Suppose $\mathcal{B}_{k}(S) \subseteq \mathcal{B}_{k}\left(\Sigma_{2 r+1}\right)$ for $k>0$. We have to show that $\mathcal{B}_{k+1}(S) \subseteq \mathcal{B}_{k+1}\left(\Sigma_{2 r+1}\right)$.

Since the radius of the CA is $r$, the set of blocks $\mathcal{B}_{k+1}\left(\Sigma_{2 r+1}\right)$ is completely determined by the set of blocks $\mathcal{B}_{k}\left(\Sigma_{4 r+1}\right)$ as well as the set of blocks $\mathcal{B}_{k+1}\left(\Phi_{[r+1,3 r+1]}\left(S_{(F, 2 r+1)}\right)\right)$ is completely determined by the set of blocks $\mathcal{B}_{k}\left(S_{(F, 2 r+1)}\right)$. Thus, showing that $\mathcal{B}_{k}\left(S_{(F, 2 r+1)}\right) \subseteq \mathcal{B}_{k}\left(\Sigma_{4 r+1}\right)$ we can reach the conclusion $\mathcal{B}_{k+1}(S) \subseteq \mathcal{B}_{k+1}\left(\Sigma_{2 r+1}\right)$.

Let $x \in \mathcal{B}_{k}\left(S_{(F, 2 r+1)}\right)$. Since $S$ is $F$-extendible, there exist $x_{1}, . ., x_{2 r+1} \in$ $\mathcal{B}_{k}(S)$ such that $x=x_{1} \odot \ldots \odot x_{2 r+1}$. By inductive hypothesis, $x_{1}, \ldots, x_{2 r+1} \in$ $\mathcal{B}_{k}\left(\Sigma_{2 r+1}\right)$ then, by Lemma $1, x \in \mathcal{B}_{k}\left(\Sigma_{4 r+1}\right)$. 
Proposition 4. Let $\left(A^{\mathbb{Z}}, F\right)$ be a $C A$ with radius $r$ and let $S \subseteq\left(A^{2 r+1}\right)^{\mathbb{N}}$ be a sofic shift. Then it is decidable if $S$ is $F$-extendible.

Proof. Given a labeled graph representation of $S$, it is possible to compute $S_{(F, 2)}$ and it is possible to compute labeled graph representations for $\Phi_{[1,2 r+1]}\left(S_{(F, 2)}\right)$ and $\Phi_{[2,2 r+2]}\left(S_{(F, 2)}\right)$. Given labeled graph representation of $S, S^{\prime}=\Phi_{[1,2 r+1]}\left(S_{(F, 2)}\right)$ and $S^{\prime \prime}=\Phi_{[2,2 r+2]}\left(S_{(F, 2)}\right)$ it is easy to build three finite state automata whose recognized languages are respectively $\mathcal{L}(S), \mathcal{L}\left(S^{\prime}\right)$ and $\mathcal{L}\left(S^{\prime \prime}\right)$. Then, the proof follows from Proposition 2 and from the decidability of the equivalence between finite state automata.

Proposition 5. Let $\left(A^{\mathbb{Z}}, F\right)$ be a $C A$ with radius $r$ and let $S \subseteq \Sigma_{2 r+1}$ be a sofic shift. Then it is decidable if $\Sigma_{2 r+1}=S$.

Proof. We provide a proof for the following claim which trivially is algorithmically checkable.

Let $M=\left(Q, A^{2 r+1}, q_{0}, F, \delta\right)$ be the smallest $D F A$ recognizing the language $\mathcal{L}(S)$. Let $N=\left(|Q| \cdot|A|^{2 r+1}\right)^{2 r+1}$. Then $\Sigma_{2 r+1}=S$ if and only if $\mathcal{B}_{N}\left(\Sigma_{4 r+1}\right)=\mathcal{B}_{N}\left(S_{(F, 2 r+1)}\right)$.

By Lemma 3, the necessary condition is trivially true. Obviously, if $\Sigma_{4 r+1}=$ $S_{(F, 2 r+1)}$ then $\Sigma_{2 r+1}=S$. Thus, we show by induction on $k>0$ that $\mathcal{B}_{k}\left(\Sigma_{4 r+1}\right)=\mathcal{B}_{k}\left(S_{(F, 2 r+1)}\right)$.

a. (Base Case) By hypothesis, $\mathcal{B}_{N}\left(\Sigma_{4 r+1}\right)=\mathcal{B}_{N}\left(S_{(F, 2 r+1)}\right)$. Moreover, since the language of a subshift is factorial, $\mathcal{B}_{k}\left(\Sigma_{4 r+1}\right)=\mathcal{B}_{k}\left(S_{(F, 2 r+1)}\right), \forall k \leq N$.

b. (Inductive Case) Suppose $\mathcal{B}_{K}\left(\Sigma_{4 r+1}\right)=\mathcal{B}_{K}\left(S_{(F, 2 r+1)}\right), K \geq N$. We have to show that $\mathcal{B}_{K+1}\left(\Sigma_{4 r+1}\right)=\mathcal{B}_{K+1}\left(S_{(F, 2 r+1)}\right)$.

Let $\mathcal{G}=(V, E, \zeta)$ be the labeled graph presentation of $S$ derived from the smallest DFA $M$ according to the procedure described at the end of section 2.1. Note that the number of vertices of $\mathcal{G}$ is less then or equal to $|Q| \cdot|A|^{2 r+1}$. Moreover, let $\mathcal{G}_{(F, 2 r+1)}$ be the $(F, 2 r+1)$-extension of $\mathcal{G}$. Note that the number of vertices of $\mathcal{G}_{(F, 2 r+1)}$ is less then or equal to $N$.

Let $a \in \mathcal{B}_{K+1}\left(\Sigma_{4 r+1}\right)$ and let $a^{1}, \ldots, a^{2 r+1} \in \mathcal{B}_{K+1}\left(\Sigma_{2 r+1}\right)$ such that $a=$ $a^{1} \odot \ldots \odot a^{2 r+1}$. Since, by inductive hypothesis, $\mathcal{B}_{K}\left(\Sigma_{4 r+1}\right)=\mathcal{B}_{K}\left(S_{(F, 2 r+1)}\right)$, it follows that $\mathcal{B}_{K+1}\left(\Sigma_{2 r+1}\right)=\mathcal{B}_{K+1}(S)$ and, trivially, that $a^{1}, \ldots, a^{2 r+1} \in$ $\mathcal{B}_{K+1}(S)$. Then there exist uniques legal paths

$$
u_{1}^{1} \rightarrow \ldots \rightarrow u_{K+1}^{1}, \ldots, u_{1}^{2 r+1} \rightarrow \ldots \rightarrow u_{K+1}^{2 r+1}
$$

in $\mathcal{G}$, where $u_{1}^{i}=\left(q_{0}, a_{1}^{i}\right)$ and $\zeta\left(u_{k}^{i}\right)=a_{k}^{i}, \forall i \in[1,2 r+1], 1 \leq k \leq K+1$.

We show that there exists $x \in S_{(F, 2 r+1)}$ such that $x_{[0, K]}=a$. Let $y \in$ $S_{(F, 2 r+1)}$ such that $y_{[0, K-1]}=a_{[0, K-1]}$. One such $y$ exists since, by inductive hypothesis, $\mathcal{B}_{K}\left(\Sigma_{4 r+1}\right)=\mathcal{B}_{K}\left(S_{(F, 2 r+1)}\right)$. Then there exists an unique path $v_{0} \rightarrow v_{1} \rightarrow$.. in $\mathcal{G}_{(F, 2 r+1)}$ such that $\zeta_{2 r+1}\left(v_{i}\right)=y_{i}, i \in \mathbb{N}$ and such that $v_{0}=\left(\left(q_{0}, c_{[1,2 r+1]}\right), \ldots,\left(q_{0}, c_{[2 r+1,4 r+1]}\right)\right)$ where $c=y_{0} \in A^{4 r+1}$. Since $K>N$ there exist $0 \leq i<j<K$ such that $v_{i}=v_{j}$. Then, 
let consider $\bar{a}^{k}=a_{1}^{k} a_{2}^{k} . a_{i}^{k} a_{j+1}^{k} a_{j+2}^{k} \ldots a_{K+1}^{k}, 1 \leq k \leq 2 r+1$. Obviously, $\bar{a}^{k} \in \mathcal{L}(S) \cap \mathcal{L}\left(\Sigma_{2 r+1}\right), 1 \leq k \leq 2 r+1$. Moreover, $\bar{a}^{k}$ are compatible then, by Lemma $1, \bar{a}=\bar{a}^{1} \odot \ldots \odot \bar{a}^{2 r+1} \in \mathcal{L}\left(\Sigma_{4 r+1}\right)$ and, by inductive hypothesis, $\bar{a} \in \mathcal{L}\left(S_{(F, 2 r+1)}\right)$.

Let $l=|\bar{a}|$. Let $z \in S_{(F, 2 r+1)}$ such that $z_{(0, l)}=\bar{a}$. Then, there exists an unique path $v_{0}^{\prime} \rightarrow v_{1}^{\prime} \rightarrow$.. in $\mathcal{G}_{(F, 2 r+1)}$ such that $\zeta_{2 r+1}\left(v_{i}^{\prime}\right)=z_{i}, i \in \mathbb{N}$ and such that $v_{0}^{\prime}=v_{0}$. Moreover, since $v_{0}^{\prime}=v_{0}$ and $z_{[0, l)}=\bar{a}$, it follows that $v_{k}^{\prime}=v_{k}$ for $0 \leq k \leq i$ and $v_{i+k}^{\prime}=v_{j+k}, 1 \leq k \leq C$ where $C=K-j$. Then it is easy to see that

$$
v_{0} \rightarrow \ldots \rightarrow v_{K} \rightarrow v_{i+C+1}^{\prime} \rightarrow v_{i+C+2}^{\prime} \rightarrow \ldots
$$

is a legal path in $\mathcal{G}(F, 2 r+1)$ and that the labelings of the vertices in the path generate a sequence $x \in S_{(F, 2 r+1)}$ such that $x_{[0, K]}=a$.

Now we are ready to state our main result and next to show the most immediate consequences.

Theorem 3. Let $\left(A^{\mathbb{Z}}, F\right)$ be a $C A$ with radius $r$ and let $S \subseteq\left(A^{2 r+1}\right)^{\mathbb{N}}$ be a sofic shift. Then it is decidable if $S=\Sigma_{2 r+1}$.

Proof. $S=\Sigma_{2 r+1}$ if and only if $S$ is $F$-extendible and $S \supseteq \Sigma_{2 r+1}$. Then, the proof follows from Proposition 4 and Proposition 5.

We now explore some important consequences of Theorem 3 related to regular CA.

Theorem 4. Let $\left(A^{\mathbb{Z}}, F\right)$ be a regular $C A$. Then $\forall t>0, \Sigma_{t}$ is computable.

Proof. Let $r$ be the radius of the CA. By Theorem 3, given a sofic shift $S \subseteq\left(A^{2 r+1}\right)^{\mathbb{N}}$, it is possible to decide if $S=\Sigma_{2 r+1}$. We can enumerate all labeled graph representing all sofic shifts contained in $A^{2 r+1}$. Then there exists an algorithm that iteratively generates graphs in the enumeration and checks if the shift represented is $\Sigma_{2 r+1}$. Since $\left(A^{\mathbb{Z}}, F\right)$ is regular, $\Sigma_{2 r+1}$ will be eventually generated and recognized. This proves that, if $\left(A^{\mathbb{Z}}, F\right)$ is regular, $\Sigma_{2 r+1}$ is computable.

In general, if $t<2 r+1$, we can compute $\Sigma_{t}$ by simply taking the projection $\Phi_{[1, t]}\left(\Sigma_{2 r+1}\right)$ otherwise, if $t>2 r+1$, by Lemma 3 point $b$, we can compute $\Sigma_{t}$ by computing the $(F, t-2 r)$-extension of $\Sigma_{2 r+1}$.

The following theorem gives an answer to a question raised in [3].

Theorem 5. The topological entropy of one-sided regular CA is computable.

Proof. Since the entropy of sofic shifts is computable, the conclusion follows from Theorem 1 and Theorem 4.

The following theorem shows that if we restrict to the class of regular CA, it is possible to provide answers to questions which are undecidable in the general case. 
Theorem 6. Let $\left(A^{\mathbb{Z}}, F\right)$ be a regular $C A$. Then the following topological properties are decidable.

1. Nilpotency

2. Equicontinuity

3. Positively Expansiveness

Proof. By Theorem 4, given $\left(A^{\mathbb{Z}}, F\right)$, it is possible to compute $\Sigma_{2 r+1}$.

1. It is easy to see that $\left(A^{\mathbb{Z}}, F\right)$ is nilpotent if and only if there exists $a \in A^{2 r+1}$ and $N>0$ such that $\forall n \geq N, \forall x \in \Sigma_{2 r+1}, \sigma^{n}(x)=a$. Given a labeled graph representation of $\Sigma_{2 r+1}$, this last condition is trivially algorithmically checkable.

2. It is easy to see that $\left(A^{\mathbb{Z}}, F\right)$ is equicontinuous if and only if $\mathcal{L}\left(\Sigma_{2 r+1}\right)$ is a bounded periodic language and that, given a labeled graph representation of $\Sigma_{2 r+1}$, it is algorithmically checkable if $\mathcal{L}\left(\Sigma_{2 r+1}\right)$ is bounded periodic.

3. Every positively expansive CA is conjugated to $\left(\Sigma_{2 r+1}, \sigma\right)$ where $\Sigma_{2 r+1}$ is a shift of finite type and, in particular, it is an $n$-full shift (see [12]). Since, for positively expansive CA, $n=\left|F^{-1}(x)\right|$ for every $x \in A^{\mathbb{Z}}, n$ is a computable number. The proof follows from the decidability of the conjugacy problem for one-sided shifts of finite type (see [15]).

To conclude, we show that, as a negative consequence of the decidability of properties in Theorem 6 , regularity is an undecidable property which implies that the membership in Kurka's language classes is undecidable.

Theorem 7. It is undecidable whether a $C A$ is regular.

Proof. Assume it is decidable if a CA is regular. Then, since nilpotent CA are regular, by Theorem 6 , it is possible to decide if a CA is nilpotent.

\section{Conclusions and open problems}

We investigated decidable properties for regular cellular automata. We showed that regularity itself is not a decidable property (Theorem 7) and that, conversely, for regular cellular automata nilpotency, equicontinuity and positively expansiveness are decidable properties (Theorem 6). Moreover we aswered a question raised in [3] showing that the topological entropy is computable for one-sided regular CA (Theorem 5). It is unknown if almost equicontinuity and sensitivity are or not decidable properties for regular CA (since to be almost equicontinuous or sensitive is a dicotomy for CA, this two properties are either both decidable or both not decidable).

\section{References}

1. F. Blanchard, A. Maass. Dynamical Behaviour of Coven's Aperiodic Cellular Automata. Theor. Comput. Sci., 163, 291-302 (1996). 
2. F. Blanchard, A. Maass. Dynamical properties of expansive one-sided cellular automata. Israel J. Math. 99, 149-174 (1997).

3. P. Di Lena. On Computing the Topological Entropy of one-sided Cellular Automata. International Journal of Unconventional Computing (1995). To appear.

4. B.Durand, E.Formenti, G. Varouchas. On undecidability of equicontinuity classification for cellular automata. Discrete models for complex systems, DMCS '03 (Lyon), 117-127 (2003).

5. R.H. Gilman, Robert H. Classes of linear automata. Ergodic Theory Dynam. Systems 7, no. 1, 105-118 (1987).

6. R.H. Gilman. Notes on Cellular Automata. Preprint (1988).

7. Hedlund, G. A. Endormorphisms and automorphisms of the shift dynamical system. Math. Systems Theory 3, 320-375 (1969).

8. L.P. Hurd, J. Kari, K. Culik. The topological entropy of cellular automata is uncomputable. Ergodic Theory Dynam. Sys. 12, no. 2, 255-265 (1992).

9. J. Hopcroft, J.D. Ullman. Introduction to automata theory, languages, and computation. Addison-Wesley Series in Computer Science. Addison-Wesley Publishing Co., Reading, Mass. (1979).

10. Z.S. Jiang, H.M. Xie. Evolution complexity of the elementary cellular automaton rule 18. Complex Systems 13, no. 3, 271-295 (2001).

11. J. Kari. The nilpotency problem of one-dimensional cellular automata. SIAM J. Comput. 21, no. 3, 571-586 (1992).

12. P. Kůrka. Topological and symbolic dynamics. Cours Spécialisés [Specialized Courses], 11. Société Mathématique de France, Paris (2003).

13. P. Kůrka. Languages, equicontinuity and attractors in cellular automata. Ergodic Theory Dynamical Systems 17, no. 2, 417-433 (1997).

14. P. Kůrka. Zero-dimensional dynamical systems, formal languages, and universality. Theory Comput. Syst. 32, no. 4, 423-433 (1999).

15. D. Lind, B. Marcus. An introduction to symbolic dynamics and coding. Cambridge University Press, Cambridge (1995).

16. J. von Neumann. Theory of self-reproducing automata. Univ. of Illinois Press, Urbana (1966). 